ISSN-PRINT 1794-9831 / E-ISSN 2322-7028

Vol. 18 N 2 / Mayo - Agosto, 2021 / Cúcuta, Colombia.

doi https://doi.org/10.22463/17949831.2763

Original

\title{
Necesidades de cuidado y diagnósticos de enfermería en gestantes con depresión: estudio descriptivo
}

\section{Care needs and nursing diagnoses of expectant mothers with depression: descriptive study Necessidades de cuidado e diagnósticos de enfermagem em gestantes com depressão: estudo descritivo}

\author{
Johanna Londoño-Restrepo ${ }^{1}$ \\ Matilde Ligia Álvarez-Gómez ${ }^{2}$ \\ Yolanda Restrepo-Suarez ${ }^{3}$ \\ Laura Daniela Londoño-Galviz ${ }^{4}$ \\ Daniela Mira-Restrepo ${ }^{5}$
}

\section{Resumen}

Objetivo: describir las necesidades y los diagnósticos de enfermería en gestantes con depresión. Materiales y método: estudio descriptivo, retrospectivo, transversal. La muestra no probabilística a conveniencia, estuvo constituida por 55 gestantes con depresión, hospitalizadas entre enero 2013 a diciembre 2018. La información se recolectó a través de la aplicación de dos cuestionarios: el primero se construyó teniendo en cuenta los objetivos y la operacionalización de variables relacionadas con información demográfica, obstétrica y psiquiátrica ; el segundo con las necesidades de cuidado, tomadas del manual de valoración de M Gordon y sintomatología del DSM_5. Se extrajeron frecuencias absolutas y relativas para las variables cualitativas, mediana y RIC para la edad. Resultados: los diagnósticos de enfermería más frecuentemente formulados fueron los siguientes: deterioro de la regulación del estado de ánimo un $67,3 \%$; riesgo de suicidio un $56,4 \%$; desesperanza con un 52,7\%; dolor agudo un $50,9 \%$; insomnio un $50,9 \%$. Las necesidades de cuidado más frecuentes fueron: cambios en el estado de ánimo un $67.3 \%$; conducta suicida un $56.4 \%$; visión negativa de sí misma y del futuro un $52.7 \%$; dolor un $50.9 \%$ e insomnio un $50.9 \%$. Conclusiones: según los patrones alternados, las necesidades de cuidado y los diagnósticos, el componente de la salud más afectado en estas mujeres fue el mental; se encontró que estas pacientes tienen mayor frecuencia de consumo de drogas, menos adherencia al tratamiento psiquiátrico y más actitudes relacionadas con: conductas suicidas, desesperanza, visión negativa del futuro y el dolor, que en otros estudios en gestantes y en diagnósticos de enfermería con depresión.

Palabras clave: Atención de enfermería, cuidado, salud mental, diagnóstico de enfermería, depresión, mujeres embarazadas.
Autor de correspondencia*

${ }^{1 *}$ Enfermera, Magíster en Epidemiología, docente, Facultad de Enfermería, Universidad de Antioquia. Medellín, Colombia. Correo: johana.londono@udea.edu.co (D) 0000-00019866-227X

${ }^{2}$ Enfermera, Especialista en Farmacodependencia, docente, Facultad de Enfermería, Universidad de Antioquia. Medellín, Colombia: Correo: matilde.alvarez@udea.edu.co (ID) 0000-0003-2093-898X

${ }^{3}$ Enfermera, Especialista en Farmacodependencia, docente, Facultad de Enfermería, Universidad de Antioquia. Medellín, Colombia. Correo: delacruz.restrepo@udea.edu.co (D) 0000-0003-3075-7832

${ }^{4}$ Estudiantes de Enfermería, Facultad de Enfermería, Universidad de Antioquia. Medellín, Colombia. Correo: laurad.londono@ $\underline{\text { udea.edu.co (iD) 0000-0003-1445-4444 }}$

${ }^{5}$ Estudiantes de Enfermería, Facultad de Enfermería, Universidad de Antioquia. Medellín, Colombia. Correo: daniela.mirar@ $\underline{\text { udea.edu.co (iD) 0000-0002-0852-2220 }}$

Recibido: 20 octubre 2020

Aprobado: 12 abril 2021

Para citar este artículo / To reference this article / Para citar este artigo:

Londoño-Restrepo J, Álvarez-Gómez ML, Restrepo-Suarez Y, Londoño-Galvis LD, Mira-Restrepo D. Necesidades de cuidado y diagnósticos de enfermería en gestantes con depresión: estudio descriptivo. Rev. cienc. cuidad. 2021; 18(2):33-42. https://doi. org/10.22463/17949831.2763

(C) Universidad Francisco de Paula Santander. Este es un artículo bajo la licencia CC-BY$\mathrm{NC}-\mathrm{ND}$ variables related to the demographic, obstetrics, and psychiatric information; the second one with the care needs, taken from the M. Gordon's assessment guideline and symptomatology 


\section{Scientific Journal of Nursing}

from DSM-5. Absolute and relative frequencies were extracted from the qualitative variables, median and IQR for age. Results: The most frequent nursing diagnoses were the following: deterioration of mood regulation $67.3 \%$; suicide risk $56.4 \%$; hopelessness $52.7 \%$; acute pain $50.9 \%$; insomnia $50.9 \%$. The most frequent care needs were: mood swings $67.3 \%$; suicidal behavior 56.4\%; negative perspective of one-self and the future $52.7 \%$; pain $50.9 \%$. Conclusions: According to alteration patterns, care needs and the diagnoses, the mental component was the most affected health component for these women; it was found that the patients have a high tendency of drug consumption, unwillingness to take the psychiatric treatment and more attitudes related to: suicidal behavior, hopelessness, a negative perspective of the future, and pain, showing that depression is highly present in expectant mothers.

Keywords: Nursing care, care, mental health, nursing diagnoses, depression, expectant mothers.

\section{Resumo}

Objetivo: descrever as necessidades e os diagnósticos de enfermagem em gestantes com depressão. Materiais e métodos: estudo descritivo, retrospectivo, transversal. A amostra não probabilística a conveniência esteve formada por 55 mulheres grávidas com depressão, internadas entre janeiro de 2013 e dezembro de 2018. A informação coletou-se através da aplicação de dois formulários: o primeiro, construiu-se considerando os objetivos e a operacionalização das variáveis relacionadas com a informação demográfica, obstétrica e psiquiátrica; o segundo com as necessidades de cuidado, tomadas do manual de valoração de M Gordon e sintomatologia do DSM-5. Obtiveram-se frequências absolutas e relativas para as variáveis qualitativas, mediana e RIQ para a idade. Resultados: os diagnósticos de enfermagem mais frequentemente formulados foram: deterioro da regulação do estado de animo $(67,3 \%)$; risco de suicídio $(56,4 \%)$; desesperança $(52,7 \%)$; dor aguda $(50,9 \%)$ e insônia $(50,9 \%)$. As necessidades de cuidado mais frequentes foram: mudanças no estado de animo $(67,3 \%)$; conduta suicida (56,4\%); visão negativa de si mesmo e do futuro $(52,7 \%)$ e insônia $(50.9 \%)$ Conclusões: segundo os padrões de alteração, as necessidades de cuidado e os diagnósticos , o componente de saúde mais afetado nessas mulheres foi o mental, encontrando-se que essas pacientes têm uma grande tendência ao consumo de drogas, menos disposição ao tratamento psiquiátrico e mais atitudes relacionadas com condutas suicidas, desesperança, visão negativa do futuro e dor, observando-se que nessas mulheres grávidas está presente a depressão.

Palavras-chave: Cuidados de enfermagem, cuidado, saúde mental, diagnóstico de enfermagem, depressão, gestantes.

\section{Introducción}

Los trastornos depresivos hacen referencia a un grupo de enfermedades mentales que se caracterizan por: la disminución del estado de ánimo, pérdida de la capacidad para disfrutar, perdida de interés en el trabajo, sentimientos de minusvalía, insomnio, anorexia e ideas suicidas, a menudo acompañados de síntomas fisiológicos, cognoscitivos y de ansiedad (1).

La depresión en gestantes es el trastorno mental más común en ese periodo; la prevalencia de depresión en gestantes va $29 \%$ en Suramérica (2) hasta $16,66 \%$ en embarazadas de México (3); porcentaje alto si se compara con la prevalencia de depresión en población general colombiana que es de 1,6 (4). Aproximadamente 


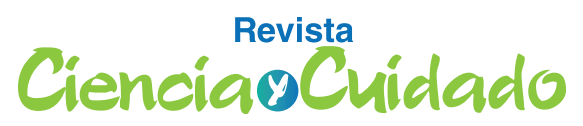

Scientific Journal of Nursing
Johanna Londoño-Restrepo, Matilde Ligia Álvarez-Gómez,Yolanda Restrepo-Suarez, Laura Daniela Londoño-Galviz.

Daniela Mira-Restrepo. más de la mitad, es decir, un $61,4 \%$ de las gestantes hospitalizadas en servicios de alto riesgo tienen síntomas depresivos (5).

Conviene indicar que los trastornos depresivos acarrean consecuencias negativas en las mujeres, el feto y el niño; las gestantes con depresión, sufren alteraciones del sueño, anhedonia, anorexia, estrés e ideas suicidas. Razón por la cual, los hijos de estas mujeres a menudo tienen problemas de sueño, retardo cognoscitivo y psicomotor, hiperactividad, depresión, ansiedad y conductas violentas (2).

Con el propósito de indagar sobre la situación de las personas con trastornos mentales se han realizado investigaciones basadas en la valoración por patrones funcionales de Marjory Gordon (6) y en la formulación de diagnósticos de enfermería. A partir de la revisión de literatura, un estudio en personas con trastorno bipolar, encontró que los patrones mas comprometidos fueron: percepción de la salud; nutrición y metabolismo; actividad y ejercicio; sueño- descanso; cognitivo, perceptivo, autopercepción y autoconcepto. Las necesidades de cuidado más comunes fueron: carencia de introspección $77,8 \%$; insomnio 57,6\%; alucinaciones o ilusiones 43,7\%. Los diagnósticos de enfermería fueron: gestión ineficaz de la salud; trastorno del patrón del sueño; desequilibrio nutricional, inferior a las necesidades corporales; y, deterioro en la toma de decisiones independiente (7).

Otra investigación en niños y adolescentes con intento de suicidio describió que los patrones más afectados y la necesidad de cuidado respectiva fueron: autopercepción, autoconcepto y cambios en el estado de ánimo $75 \%$, seguido del aburrimiento $37,5 \%$ y no sentirse bien consigo mismo. Del Patrón de Tolerancia al Estrés, problemas familiares $68,8 \%$ y fallecimiento de un miembro de la familia $50 \%$. Con respecto al patrón ognitivo perceptivo se señalaron: antecedentes familiares con intento de suicidio en un 50\%, exposición a venenos un $75 \%$ y consumo de alcohol un 33\%. En cuanto al patrón de sueño y descanso, el 50\% presentó alteración de este patrón; además, un $25 \%$ con aumento del sueño (8).

Por otra parte, se encontraron dos estudios sobre personas con depresión. Uno cuya fuente de información correspondió a 12 enfermeras que cuidaban pacientes deprimidos en Atenas, quienes formularon a 11 personas el diagnóstico riesgo de suicidio, a 10 aislamiento social y nutrición alterada y a 9 déficit de autocuidado (9). El otro estudio formuló los siguientes diagnósticos de enfermería: baja autoestima, insomnio, desesperanza y riesgo de suicidio (10).
Como se mencionó, los trastornos depresivos repercuten de forma negativa en la gestante, el feto y el niño; es por esto que se han formulado una serie de diagnósticos de enfermería que buscan fortalecer el cuidado en la salud mental materno perinatal. En función de lo planteado, con el desarrollo de esta investigación se da cumplimento al objetivo general y específicos, que se formulan a continuación.

\section{Objetivos}

\section{Objetivo general}

Describir los diagnósticos de enfermería en gestantes con diagnóstico psiquiátrico de depresión.

\section{Objetivos específicos}

- Describir las características demográficas, obstétricas y psiquiátricas en gestantes con depresión; identificar las necesidades de cuidado, los patrones funcionales alterados y los diagnósticos de enfermería.

- En definitiva, con el desarrollo de los objetivos se construirán los planes de cuidado que servirán de orientación para el personal de enfermería; igualmente, se fortalecerá la calidad del cuidado y se brindarán herramientas para el desarrollo de competencias en la formación de enfermería en el área de salud mental perinatal.

\section{Materiales y Métodos}

Se realizó un estudio descriptivo, retrospectivo y transversal. La muestra no probabilística a conveniencia, estuvo constituida por 55 historias clínicas, que correspondieron a la totalidad de gestantes con depresión, hospitalizadas en un servicio de ARO (Alto Riesgo Obstétrico) de una clínica de Medellín (Colombia), entre enero de 2013 a diciembre de 2018. Estas gestantes son remitidas para hospitalización por causa del ARO, en el Área Metropolitana del Valle de Aburrá, que comprende los municipios de: Medellín, Envigado, Itagüí, Bello y Copacabana. Las pacientes pertenecen en su mayoría al sistema privado de seguridad social.

Se incluyeron las historias completas de gestantes de cualquier edad en la primera hospitalización con uno de los siguientes trastornos depresivos: episodio depresivo leve, moderado, severo sin síntomas psicóticos, distimia, otros episodios depresivos, episodio depresivo persistente, episodio depresivo único y otros trastornos depresivos no especificados. Se excluyeron 44 historias clínicas de gestantes con episodio depresivo grave, 
con síntomas psicóticos y con historias que tenían datos incompletos.

Para la recolección de la información se construyó un instrumento tipo cuestionario, que se aplicó a las 55 historias clínicas de las embarazadas con diagnóstico de depresión. Dicho cuestionario tuvo dos partes: la primera estuvo conformada por los ítems que indagaban sobre la información demográfica, obstétrica y mental; $\mathrm{y}$, la segunda por una lista de chequeo que contenía 116 necesidades de cuidado, distribuidas por patrones funcionales. Estas necesidades se extrajeron de la sintomatología mental del inventario de depresión de Beck (11); de los criterios diagnósticos del DSM- 5 (Diagnostic and Statistical Manual of Mental Disorders) (12); y, del Manual de Valoración de Marjory Gordon (6).

Para asegurar la confiabilidad, validez y disminución de sesgos, se formularon varias preguntas sobre cada uno de los patrones funcionales; luego fue sometido a evaluación por dos expertas en enfermería psiquiátrica y proceso de enfermería. También, se realizó una prueba piloto, que fue corregida; además, se estandarizaron los investigadores para la recolección de la información y registro de los datos en el cuestionario.

La información se obtuvo de la primera nota de valoración realizada por psiquiatras, obstetras, enfermeras, trabajadores sociales y psicólogos. Una vez recolectada se elaboró una base de datos que se procesó en el programa SPSS versión 25. A continuación, se realizó un análisis univariado, se calcularon las frecuencias absolutas y relativas para las variables cualitativas; respecto de la edad que fue la única variable cuantitativa se extrajo de la mediana y el RIC. Para formular los diagnósticos, se tuvieron en cuenta las necesidades más comunes y se introdujeron en el NNN Consult; asimismo, se escogieron los diagnósticos, según características definitorias.

Esta investigación fue aprobada por el Comité de Ética de la Facultad de Enfermería de la Universidad de Antioquia (acta CEI-FE 2019-17) y el Comité de Ética de salud (acta N15) de donde se recolectó la información. Se consideró sin riesgo para la salud según Resolución 8430 de 1993, emanada del Ministerio de Salud de la República de Colombia, la declaración de Helsinki 2016 y la declaración CIOMS 2016 (13).

\section{Resultados}

\section{Características demográficas}

La mediana de edad fue de 26 años (RIC 12), la escolaridad más frecuente correspondió a la secundaria con $22(40 \%)$, seguida por la formación superior 13 $(23,6 \%)$. Según el sitio de residencia, la más frecuente fue la urbana con $48(87,3 \%)$ y en situación de calle se encontraron $2(3,6 \%)$ de las pacientes. Según ingreso económico, $54(98,2 \%)$ devengaban menos de dos salarios mínimos. Las demás características se aprecian en la Tabla 1.

Tabla 1. Características demográficas de 55 gestantes con depresión, hospitalizadas en alto riesgo obstétrico, en una clínica privada de Medellín.

\begin{tabular}{ccc}
\hline Característica & Frecuencia (n) & \% \\
\hline Estado civil & & \\
$\quad$ Soltera & 26 & 47,3 \\
Casada /unión libre & 28 & 50,9 \\
Ocupación & & \\
Ama de casa & 27 & 49,1 \\
Empleada / trabajadora independiente & 15 & 27,2 \\
Estudiante & 2 & 3,6 \\
Desempleada & 7 & 12,7 \\
Seguridad social & & \\
Contributivo & 43 & 78,2 \\
Subsidiado & 11 & 21,8 \\
\hline
\end{tabular}

Fuente: Elaboración propia de las investigadoras 


\section{CienciageCuidado}

Scientific Journal of Nursing

\section{Características Obstétricas}

Con relación a las semanas de gestación, se encontró que $38(69,1 \%)$ estaban en tercer trimestre y 14 $(25,5 \%)$ en el segundo. Según la paridad: 22 (40\%) son primigestantes, $10(18,2 \%)$ segundigestantes y 15 $(27,3 \%)$ multigestantes. De las 33 gestantes que tienen hijos, $13(39,4 \%)$ la vía del parto fue por cesárea; de la totalidad de gestantes $38(30,9 \%)$ tenían antecedentes de aborto.
La principal causa de ARO se relacionó con: amenaza de parto pretérmino $16(29,1 \%)$; seguido de depresión $10(18,2 \%)$; farmacodependencia $8(14,5 \%)$; diabetes gestacional e hipertensión arterial $7(12,7 \%)$ respectivamente; e intento de suicidio un $5(9,1 \%)$. Otras causas de ARO de menor frecuencia fueron: ruptura prematura de membranas, hiperémesis gravídica y cardiopatía.

\section{Características psiquiátricas}

Tabla 2. Tipo de episodio depresivo en 55 gestantes de ARO, de alto riesgo obstétrico, en una clínica privada de Medellín.

\begin{tabular}{ccc}
\hline Tipo de episodio & Frecuencia (n) & $\mathbf{\%}$ \\
\hline Episodio depresivo grave & 6 & 10,9 \\
Otros episodios depresivos (ansie- & 5 & 9,1 \\
dad, somatización) & & \\
Distimia & 4 & 7,3 \\
Otros episodios no específicos & 4 & 7,3 \\
\hline
\end{tabular}

Fuente: Elaboración propia de las investigadoras

El tipo de depresión más frecuente fue el episodio depresivo moderado $35(63,6 \%)$, otros datos se pueden apreciar en la Tabla 2.

De los antecedentes psiquiátricos el más frecuente fue haber sufrido de depresión 40 (80\%), seguido de antecedentes de consumo de sustancias psicoactivas incluidos el alcohol y el tabaco $13(23,6 \%)$; también se presentaron: ansiedad $12(21,8 \%)$ y ser diagnosticada con depresión posparto $6(10,9 \%)$. Se resalta que el $18,2 \%$ fue víctima de algún tipo de maltrato.

\section{Necesidades y diagnósticos de enfermería}

Los patrones funcionales alterados fueron: el Cognitivo Perceptivo 53 (96,4\%); de Afrontamiento y Tolerancia al Estrés $46(83,6 \%)$; Autopercepción y Autoconcepto 43 (78,2\%); Percepción y Manejo de la Salud 35 (63,6\%); Nutricional Metabólico 34 (61,8\%); de Rol y Relaciones 33 (60\%); Sueño y Reposo 33 (60\%); Actividad y Ejercicio 28 (50,9\%); Sexualidad 23 (41,8\%); Eliminación 20 (36,4\%); y, de Creencias y Valores 17 $(30,9 \%)$.

Las necesidades más comunes por patrones fueron: el de Percepción y Manejo de la Salud, falta de adherencia al tratamiento psiquiátrico $11(20 \%)$; el Nutricional
Metabólico, pérdida del apetito $20(36,4 \%)$ y cambios en el peso $17(30,9 \%)$; el de Eliminación, la necesidad más frecuente fue problemas urinarios 17 (30,9\%); el de Actividad y Ejercicio, se observaron cambios en la presión arterial $12(21,8 \%)$; el de Sueño y Reposo, insomnio $28(50,9 \%)$. Además, se encontraron los siguientes efectos en el patrón Cognitivo Perceptivo: dolor 28(50,9\%), episodio de llanto $27(49,1 \%)$, carencia de introspección $23(41,8 \%)$, anhedonia 21 $(38,2 \%)$, hipotimia $16(29,1 \%)$ y sentimiento de culpa e irritabilidad 15 (27,3\%). De otra parte, en los siguientes patrones se percibieron efectos que se mencionan a continuación: Autopercepción y Autoconcepto, visión negativa del futuro 29 (52,7\%); Rol y Relaciones, problemas familiares 23 (41,8\%); de Sexualidad y Reproducción, $20(36,4 \%)$ de las pacientes presentaron alteración del bienestar fetal; Afrontamiento y Tolerancia al Estrés, 37 (67,3\%) mostraron cambios en el estado de ánimo, $31(56,4 \%)$ tuvieron ideas suicidas y/o intento suicida; $y$, valores y creencias, sufrimiento espiritual un $(9,1 \%)$.

Los diagnósticos de enfermería más comunes estuvieron relacionados con el deterioro de la regulación del estado de ánimo y riesgo de suicidio, los demás se aprecian en la Tabla 3. 


\section{Scientific Journal of Nursing}

Tabla 3. Tipo de episodio depresivo en 55 gestantes de ARO, de alto riesgo obstétrico, en una clínica privada de Medellín.

\begin{tabular}{|c|c|c|c|c|c|}
\hline \multirow[t]{2}{*}{ Patrón funcional } & \multirow{2}{*}{$\begin{array}{l}\text { Necesidades de } \\
\text { cuidado }\end{array}$} & \multirow{2}{*}{$\begin{array}{l}\text { Fre- } \\
\text { cuen- } \\
\text { cia }(n)\end{array}$} & \multirow[t]{2}{*}{$\%$} & \multicolumn{2}{|c|}{ Diagnósticos enfermeros } \\
\hline & & & & Dominio NANDA & $\begin{array}{l}\text { Etiqueta diag- } \\
\text { nóstica }\end{array}$ \\
\hline $\begin{array}{l}\text { Afrontamiento-tol- } \\
\text { erancia del estrés }\end{array}$ & $\begin{array}{l}\text { Cambios en el } \\
\text { estado de animo }\end{array}$ & 37 & 67,3 & $\begin{array}{l}\text { 9. Afrontamiento/Toler- } \\
\text { ancia al Estrés } \\
\text { Clase 2. Respuestas de } \\
\text { afrontamiento }\end{array}$ & $\begin{array}{l}\text { Deterioro de la } \\
\text { regulación del es- } \\
\text { tado de ánimo }\end{array}$ \\
\hline $\begin{array}{l}\text { Afrontamiento-tol- } \\
\text { erancia del estrés }\end{array}$ & $\begin{array}{l}\text { Intento y/o ideas } \\
\text { suicidas }\end{array}$ & 31 & 56,4 & $\begin{array}{l}\text { 11. Seguridad/Protección } \\
\text { Clase 3. Violencia }\end{array}$ & $\begin{array}{l}\text { Riesgo de suici- } \\
\text { dio }\end{array}$ \\
\hline $\begin{array}{l}\text { Autopercepción y } \\
\text { autoconcepto }\end{array}$ & $\begin{array}{l}\text { Visión negativa } \\
\text { de sí misma, del } \\
\text { futuro y falta de } \\
\text { propósito }\end{array}$ & 29 & 52,7 & $\begin{array}{l}\text { 6. Autopercepción } \\
\text { Clase 2. Autoestima }\end{array}$ & Desesperanza \\
\hline $\begin{array}{l}\text { Perceptivo- cogni- } \\
\text { tivo }\end{array}$ & Dolor & 28 & 50,9 & $\begin{array}{l}\text { 12.Confort } \\
\text { Clase 1. Confort físico }\end{array}$ & Dolor agudo \\
\hline Sueño-reposo & Insomnio & 28 & 50,9 & $\begin{array}{l}\text { 4. Actividad/Reposo } \\
\text { Clase 1. Sueño-reposo }\end{array}$ & Insomnio \\
\hline $\begin{array}{l}\text { Perceptivo-cogni- } \\
\text { tivo }\end{array}$ & $\begin{array}{l}\text { Episodios } \\
\text { llanto }\end{array}$ & 27 & 49,1 & $\begin{array}{l}\text { 5. Percepción/Cognición } \\
\text { Clase 4. Cognición }\end{array}$ & $\begin{array}{l}\text { Labilidad del con- } \\
\text { trol emocional }\end{array}$ \\
\hline
\end{tabular}

Fuente: Elaboración propia de las investigadoras

\section{Discusión}

Las características demográficas de estas gestantes son semejantes a las halladas por otros estudios sobre mujeres de ARO en Medellín $(5,14)$. Además, se encontró que las frecuencias sobre educación son mayores a los reportados en Colombia para el año 2018 e igual que los porcentajes de desempleo para este mismo año (15). Con respecto a los hallazgo obstétricos como son: estar en el tercer trimestre de la gestación, antecedentes de aborto y tener como diagnóstico de ingreso para ARO amenaza de parto prematuro, son similares a los descritos por otros investigadores (16-18).

Hay que mencionar además, que el episodio depresivo que más sufren las embarazadas es el depresivo moderado en más de la mitad; frecuencia mayor que en otro estudio realizado en Venezuela, en donde las gestantes con ARO fue de un $19,6 \%$, hecho que se puede explicar porque las nuestras tenían el diagnóstico de depresión (18).

Por otra parte, se encontró que en las gestantes prevalecían antecedentes de depresión en un $80 \%$, siendo mayor en comparación con otras investigaciones realizadas sobre los efectos de ARO en Colombia que van del 23,8\% en Medellín a un 60\% en Bucaramanga $(5,18)$. Conviene subrayar, que la frecuencia sobre antecedentes de depresión fue mayor en las mujeres objeto de este estudio, puesto que todas las gestantes tenían el diagnóstico de depresión como criterio de inclusión y se comparó con un estudio de tamizaje para síntomas depresivos y ansiosos. En este último se encontró, además, asociación entre la presencia de diagnóstico previo de depresión y sintomatología depresiva con un OR (Odds Ratio) de 7,16 (5).

De acuerdo con los demás antecedentes psiquiátricos como: ser víctimas de ansiedad, consumo de drogas y 
depresión posparto, son usualmente descritos por otros autores, indicando que los dos primeros pueden ser a su vez factores de riesgo sobre depresión en este periodo $(5,19)$. Con referencia al antecedente de depresión posparto fue menor a lo reportado por otros investigadores, cuyas frecuencias van de $14 \%$ a $16 \%$, debido a la extracción de datos de las historias clínicas; en cambio en los estudios comparados se aplicaron escalas específicas para detectar la depresión en este periodo, puesto que es un dato más preciso $(20,21)$.

Comparando con otros estudios, los porcentajes de consumo de sustancias en el que se incluyen el consumo de alcohol, tabaco y otras drogas fueron mayores en las gestantes de Medellín cuya frecuencia correspondió al $12 \%$ de alcohol en el primer trimestre y $6 \%$ de otras drogas en este mismo periodo (16). Se sabe que la depresión es comórbida con consumo de alcohol y drogas; hecho que es corroborado por un estudio realizado en Brasil, cuyo objetivo fue determinar la prevalencia de la depresión y factores de riesgo, encontrado una asociación entre depresión y consumo de sustancias psicoactivas en $7(46,7 \%) \mathrm{p}<0.001$ (22).

Haciendo alusión a los patrones funcionales se encontró que, aunque el patrón sobre Percepción de la Salud no tuvo porcentajes altos; no obstante, llama la atención que casi la cuarta parte de estas mujeres no tenían adherencia al tratamiento psiquiátrico (gestión ineficaz de la salud). En cambio, otros autores han reportado que el diagnóstico médico de depresión predispone a las personas a no aceptar el tratamiento psiquiátrico, ni al de otras enfermedades (23).

Desde otro punto de vista, un estudio sobre aceptación al tratamiento sobre depresión en pacientes ancianos, se detectó que el $13,5 \%$ no iniciaron terapia farmacológica, porcentaje menor al encontrado en el presente estudio (24).

En cuanto al patrón Nutricional Metabólico, se percibió alterado en tres cuartas partes de las pacientes, determinando que más de la cuarta parte presentaron cambios en el peso corporal y pérdida del apetito $(36,4 \%)$, síntomas que fueron señalados por criterios sobre este tipo de diagnósticos por el DSM-5 (12) para la depresión. Igualmente, otros estudios describieron alteraciones en el peso, en gestantes con trastornos mentales (25).

Con referencia al insomnio hay que precisar que es un síntoma de depresión DSM-5 (12) como un diagnóstico de enfermería; al respecto en este estudio un poco más de la mitad de las pacientes sufrían este trastorno. En un Hospital de Grecia 10 enfermeras de 12, asignaron como diagnóstico de enfermería el insomnio a pa- cientes hospitalizados con depresión; es decir, que es mayor que el hallado en este estudio (9).

Respecto al patrón Perceptivo Cognitivo se observó que es el más frecuente con un 96,4\%; aunque algunas de las necesidades de cuidado son síntomas de depresión del DSM-5 (12), tales como: la irritabilidad, la anhedonia y el llanto. Sin embargo, el dolor se manifestó en un alto porcentaje, ya que lo percibieron más de la mitad de las gestantes. Hay que aclarar que este síntoma puede encontrarse en pacientes con depresión, puesto que va acompañado de síntomas somáticos. Los dolores en personas con depresión se ubican en un 35\%, siendo los más comunes: cefalea, dolores articulares, dolores en el tórax, en miembros inferiores y abdominales (1). No obstante, en los dos últimos trimestres, las gestantes los perciben a consecuencia de contracciones de Braxton hicks, dolor suprapúbico, dolor lumbar y dolores en miembros inferiores (26).

Así mismo, en una población de adultos mayores con trastornos depresivos se advirtió que el 50\% tenía dolor. Como se puede observar los dos estudios anteriores tienen frecuencias inferiores a los hallazgos que se obtuvieron en ésta investigación (27).

Acerca del patrón de Autopercepción y Autoconcepto más de la mitad de las pacientes lo tuvieron alterado; hecho que permite concluir que necesitan de más cuidado, puesto que tienen una visión negativa de sí mismas y del futuro. En el DSM-5(12) unos de los criterios de diagnóstico para la depresión está relacionado con la desesperanza, factor que a su vez es un diagnóstico de enfermería (12). En este sentido, en un estudio realizado en Brasil, el diagnóstico de enfermería sobre la desesperanza se describió en un $57 \%$ de los pacientes con SIDA (Síndrome de Inmunodeficiencia Adquirida) (28). Así mismo, en una investigación realizada en Italia, en pacientes con depresión, el diagnóstico sobre la desesperanza estuvo presente entre el 71 y el $100 \%$ (10).

Sobre el patrón de Afrontamiento y Tolerancia al Estrés el mismo afectó al 83,6\%; demostrándose que la necesidad de cuidado más común estaba relacionado con cambios en el estado de ánimo (labilidad emocional), síntoma que se manifestó en casi las tres cuartas partes de estas mujeres. Se debe agregar, que el cambio de estado de ánimo, no es un síntoma clásico de depresión según el DSM-5(12); en cambio hace parte de la sintomatología de los trastornos de la afectividad, entre los que están los trastornos depresivos, que se pueden evaluar a través de la valoración mental (1).

Por otro lado, hay que señalar que la segunda necesidad del patrón de Afrontamiento y Tolerancia al Estrés 
se refiere a conductas suicidas (ideación e intento), que hacen parte de los criterios de diagnóstico del DSM5(12) para la depresión. En gestantes hospitalizadas se ha encontrado que de 23.000 embarazadas, 2.180 tenían conductas suicidas (29), porcentaje menor que el de este estudio. Igual sucede con los hallazgos en otros estudios realizados en América Latina, cuyas frecuencias fueron de un $35,7 \%$ en Cuba, frente al 7,2\% con ideas suicidas y el 3,6\% de intento suicida en Medellín (16,29-30).

\section{Limitaciones}

Por ser un estudio cuya fuente de información fueron las historias clínicas puede tener limitaciones debido a que todas las notas de los profesionales de salud no fueron homogéneas. El cuestionario se construyó basado en los objetivos y operacionalización de las variables, debido a que no se encontró un cuestionario validado, sobre las necesidades de cuidado en gestantes de alto riesgo. Por tal motivo, esta investigación puede servir como una oportunidad para validar dicho instrumento. Por otro parte, los datos hallados no se pueden extrapolar a todas las gestantes de $\mathrm{ARO}$, puesto que solo se realizó en una sola institución de salud de Medellín.

\section{Conclusiones}

- La información demográfica fue similar a lo reportado por otros estudios en gestantes de ARO y en mujeres de la población general colombiana, ex- ceptuando el nivel educativo que es mayor en el grupo objeto de estudio. Los hallazgos obstétricos son similares a los reportados por otros autores en población de ARO de América Latina.

- Según los patrones alterados y las necesidades de cuidado, respecto a las etiquetas diagnosticadas, el componente de salud más afectado en estas mujeres fue el mental. También, se encontró un mayor consumo de drogas, conducta suicida, desesperanza, visión negativa del futuro, dolor y menor adherencia al tratamiento psiquiátrico, que en otras investigaciones.

- Siendo el componente mental el más afectado, es necesario que los profesionales de enfermería realicen una valoración integral de la salud mental de las gestantes. De igual forma les corresponde indagar acerca de los antecedentes personales y familiares, los trastornos mentales, el consumo de sustancias psicoactivas y sintomatología depresiva. De manera análoga, hay que analizar la actitud frente al embarazo, la red de apoyo familiar y social, para establecer intervenciones que mejoren la calidad de vida, la dinámica familiar y la capacidad para afrontar situaciones adversas.

Conflicto de intereses: las autoras declaran no tener conflicto de intereses.

\section{Referencias Bibliográficas}

1. Palacio-Acosta C. Trastornos depresivos En: Palacio Acosta C, Toro Obando A. Fundamentos de Medicina: Psiquiatría. 6a Edición. Medellín: CIB (Corporación para Investigaciones Biológicas); 2018. p 152 -171

2. Martínez-Paredes JF, Jácome-Perez N. Depresión en el Embarazo. Rev Colomb Psiquiat 2019;48(1):58-65. https://doi.org/10.1016/j.rep.2017.07.003

3. Rodríguez-Baeza, A et al. Prevalencia y factores asociados a depresión prenatal en una institución de salud. Rev Enferm Inst Mex Seguro Soc. 2017;25(3):181- 188.http://revistaenfermeria.imss.gob.mx/editorial/index.php/revista enfermeria/article/view/215/400>.

4. Franco Vasquez JG, Álvarez Gomez M. Salud mental: En Uribe Rios M. Guarin Alzate G. Salud publica enfoque preventivo y practico. Editorial Pontificia Bolivariana, 2019. P. 308- 314.

5. Ricardo-Ramírez C, Álvarez-Gómez M, Ocampo-Saldarriaga MV, Tirado-Otálvaro AF. Prevalencia de tamizaje positivo para depresión y ansiedad en gestantes de alto riesgo obstétrico en una clínica de Medellín, entre enero y agosto de 2013. Factores de riesgo asociados. Rev Colomb Obstet Ginecol. 2015;66(2):94-102. https:// doi.org/10.18597/rcog.11 
6. Álvarez-Suarez J, Castillo-Arévalo F, Fernández-Fidalgo D, Muñoz-Meléndez M. Manual de valoración de patrones funcionales. Gijóon: Comisión de Cuidados. Dirección de Enfermería de Atención Primaria. Área Sanitaria V; 2010.

7. Montoya-Zapata CP, Pérez-Villa M, Rendón-Morales KJ, Quintero-Arenas AA, Urrego-Febrys IC, Álvarez-Gómez ML. Características demográficas, de salud, necesidades de cuidado y diagnósticos de enfermería de personas hospitalizadas que sufren trastorno afectivo bipolar. Investig en Enfermeria, Imagen y Desarrollo. 2019; 21(1). https://doi.org/10.11144/Javeriana.ie21-1.cdsn

8. Piedrahita LE, García MA, Mesa JS, Stivalis Rosero I. Identificación de los factores relacionados con el intento de suicidio, en niños y adolescentes a partir de la aplicación del Proceso de Atención de Enfermería. Colomb Med. 2011;42:334-341.

9. Prokofieva M, Koukia E, Dikeos D. Mental Health Nursing in Greece: Nursing Diagnoses and Interventions in Major Depression. Issues Ment Health Nurs. 2016;37(8):556-562. http://dx.doi.org/10.3109/01612840.2016.1169 $\underline{466}$

10. Prokofieva M, Karadima G, Koukia E, Michou V, Kyprianidou C, Papageorgiou CV, Alexiadis E, Constantoulakis P, Dikeos D. Association of 5-HTTLPR Polymorphism with the Nursing Diagnoses and the Achievement of Nursing Outcomes in Patients with Major Depression. Issues Ment Health Nurs. 2017;38(10):798-804. http:// doi:10.1080/01612840.2017.1364811.

11. Beck AT, Steer RA, Brown GK. Inventario de Depresión de Beck - II. Pearson; 2011.

12. Asociación Americana de Psiquiatría. DSM-5. Manual Diagnóstico y Estadístico de los Trastornos Mentales. 5a Edición. Editorial Médica Panamericana S.A; 2014.p. 103-127.

13. República de Colombia. Ministerio-de-Salud. Resolución 8430 de 1993. Bogota, Colombia; 1993.

14. Guerra AM, Dávalos-Pérez DM, Castillo-Martínez A. Detección de síntomas depresivos en mujeres gestantes de alta complejidad obstétrica y factores correlacionados. Rev Colomb Psiquiatr. 2017;46(4):215-221. http:// dx.doi.org/10.1016/j.rcp.2016.09.002

15. DANE (Departamento Administrativo Nacional de Estadística). Encuesta Nacional de Presupuestos de Hogares Colombianos [Internet]. Bogotá; 2018.

16. Vergel-Noguera J, Gaviria Arbelaez SL, Duque M, Restrepo D, Rondon M, Colonia A. Gestation-related psychosocial factors in women from Medellin, Colombia. Rev Colomb Psiquiatr. 2019;48(1):26-34. https://doi. org/10.1016/j.rcp.2017.06.003

17. Farias Pessoa da Silva G, Vitorino dos Santos S, Araújo do Nascimento JW, Silva de Santana F, Silva de Medeiros J, Brust de Jesus S. Risco de depressão e ansiedade em gestantes na atenção primária. Nursing.2020;23(271):4961-4970. $\quad$ http://revistas.mpmcomunicacao.com.br/index.php/revistanursing/article/ view/1044

18. Zambrano-Osma S, Perilla-Mojica M, Lozano-Osma MD, Redondo-Rodrigeuz S. Prevalencia de depresión $\mathrm{y}$ ansiedad y variables asociadas en gestantes de Bucaramanga y Floridablanca (Santander, Colombia). MedUNAB [Internet]. 2019;22(2):171-85. https://revistas.unab.edu.co/index.php/medunab/article/view/3586

19. Tang X, Lu Z, Hu D, Zhong X. Influencing factors for prenatal Stress, anxiety and depression in early pregnancy among women in Chongqing, China. J Affect Disord. 2019;15(253):292-302. http:// doi: 10.1016/j.jad.2019.05.003.

20. Hartmann JM, Mendoza-Sassi RA, Cesar JA. Depressão entre puérperas: prevalência e fatores associados 
Scientific Journal of Nursing

[Postpartum depression: prevalence and associated factors]. Cad Saude Publica. 2017;33(9):e00094016. http:// doi: 10.1590/0102-311X00094016.

21. Wubetu AD, Engidaw NA, Gizachew KD. Prevalence of postpartum depression and associated factors among postnatal care attendees in Debre Berhan, Ethiopia, 2018. BMC Pregnancy Childbirth. 2020;20(1):2-9. http:// doi: 10.1186/s12884-020-02873-4.

22. Silva MM de J, Leite EPRC, Nogueira DA, Clapis MJ. Depression in pregnancy. Prevalence and associated factors. Investig y Educ en Enferm. 2016;34(2):342-350. http://doi.org/10.17533/udea.iee.v34n2a14

23. Llanos-Tejada F, Ponce-Chang C. Depresión y adherencia en personas afectadas con tuberculosis. Una exploración preliminar de datos. Rev Neuropsiquiatr. 2019;82(2):104-109. http://doi.org/10.20453/rnp.v82i2.3536

24. Holvast F, Oude-Voshaar R, Wouters H, Hek K, Schellevis F, Burger H, Verhaak P. Non-adherence to antidepressants among older patients with depression: a longitudinal cohort study in primary care. Family Practice. 2019;36(1): 12-20, https://doi.org/10.1093/fampra/cmy106

25. Lucchese R, Simões ND, Monteiro LHB, Vera I, Fernandes IL, Castro PA de, et al. Factors associated with the probability of common mental disorders in pregnant women: a cross-sectional study. Esc Anna Nery. 2017;21(3):1-6. http://dx.doi.org/10.1590/2177-9465-ean-2016-0094

26. Jaramillo-Agudelo B, Restrepo O. Adaptación materna y cambios fisiológicos durante la gestación. In: Henao G, Londoño Cardona JG, Echavarría Restrepo LG, editors. Obstetricia y Ginecología. 9a Edición. Medellín: CIB (Corporacion para Investigaciones Biológicas); 2015. p. 105-113.

27. Ulbricht CM, Hunnicutt JN, Hume AL, Lapane KL. Depression, Anxiety, and Pain among Newly Admitted Nursing Home Residents. J Nurs Home Res Sci.2019; 5, 40-48. https://doi.org/10.14283/jnhrs.2019.8

28. Da Silva Richardson A, de Souza Neto V, Ribeiro Maia M, Ribeiro Barbosa A, da Silva Tavares C, de Souza Lima A e Silva Felype J. Diagnósticos de enfermagem do domínio autopercepção em pessoas vivendo com Aids. Rev Cubana Enfermer [Internet]. 2018; 34( 2 ): e1604. http://scielo.sld.cu/scielo.php?script=sci_arttext\&pid=S0864-03192018000200009\&1ng=es

29. Zhong QY, Gelaye B, Smoller JW, Avillach P, Cai T, Williams MA. Adverse obstetric outcomes during delivery hospitalizations complicated by suicidal behavior among US pregnant women. PLoS One. 2018;13(2):1-13. http://doi.org/10.1371/journal.pone.0192943

30. Machado-Solano AM, Sánchez-Hidalgo Md, Guerra-Domínguez E, Armas-Saborit C, Barrero-Machado AI. Factores de riesgo de suicidio en gestantes adolescentes. 2017-2018. RM.2018;22(5): 1005-1016. http://www. revmultimed.sld.cu/index.php/mtm/article/view/1000 